\title{
Teil F: Materialgewinnung, -lagerung und -nutzung
}

F.1. Rechtliche, insbesondere strafrechtliche Anforderungen an die Probengewinnung

$\begin{array}{lll}\text { F.2. Probenlagerung und -transport __ } & 115\end{array}$

F.3. Welchen Aufbewahrungspflichten unterliegen die Proben in einer BMB? __ 116

F.4. Haftung bei Verstößen gegen Lagerungs-, Nutzungs- und Transportbestimmungen_

F.5. Besteht ein gesetzliches Recht zur Nutzung von Biomaterialien?

F.6. Durch wen können Nutzungsrechte entzogen bzw. übertragen werden?___ 120

$\begin{array}{ll}\text { F.7. Zusammenfassung _ } 121 & 120\end{array}$ 

Hinsichtlich der rechtlichen Rahmenbedingungen von Gewinnung, Lagerung und Nutzung der Biomaterialien besteht nach Einschätzung der Gutachter kein grundsätzlicher Unterschied zwischen Materialien, die im Forschungs- oder im Behandlungszusammenhang gewonnen oder verarbeitet werden. Die einschlägigen Vorschriften sind stoff- und nicht zweckbezogen. Sofern der Spender in seiner Einwilligungserklärung Einschränkungen vorgenommen hat, müssen diese berücksichtigt werden. Außerdem ist die Zweckbestimmung einer Probe auch bei deren Lagerung und Nutzung angemessen zu berücksichtigen.

\section{F.1. Rechtliche, insbesondere strafrechtliche Anforderungen an die Probengewinnung}

\section{F.1.1. Probengewinnung}

Die Probengewinnung darf nur erfolgen, wenn hierfür eine Einwilligungserklärung des Spenders vorliegt. Ansonsten könnte der Tatbestand des \223 StGB (Körperverletzung) erfüllt sein. Dabei macht es zunächst keinen Unterschied, ob die Probe zu Forschungs- oder Behandlungszwecken entnommen wird. Allerdings reichen für Proben, die primär für die medizinische Diagnostik und Behandlung entnommen und aufbewahrt werden, der Behandlungsvertrag und die Einwilligung in den körperlichen Heileingriff aus. ${ }^{167}$ Eine gesonderte schriftliche Information und die Einwilligung des Spenders sind nur bei der Probenentnahme ausschließlich zu Forschungszwecken nötig. ${ }^{168}$

Der Arztvorbehalt gilt grundsätzlich bei invasiven ebenso wie bei nicht-invasiven Probeentnahmen. \I Heilpraktikergesetz legt fest, dass die Ausübung der Heilkunde, und dies schließt die Entnahme von Körpermaterial ein, nur durch Ärzte oder mit behördlicher Genehmigung erfolgen darf. Der Arzt kann jedoch Teile seiner Tätigkeit an Nicht-Ärzte delegieren, wenn die übertragene Tätigkeit grundsätzlich delegierbar ist. Nicht delegationsfähig sind Tätigkeiten, die aufgrund ihrer Schwierigkeit, Gefährlichkeit oder wegen der Unvorhersehbarkeit etwaiger Reaktionen ärztliches Fachwissen voraussetzen. Diese sind stets vom Arzt persönlich durchzuführen. ${ }^{169}$ Hierzu zählen insbesondere

- sämtliche operativen Eingriffe

- schwierige Injektionen

- Infusionen

- Blutentnahmen

- ärztliche Untersuchungen

- die ärztliche Aufklärung und Beratung von Patienten und Probanden.

Auch alle invasiven diagnostischen Eingriffe, insbesondere Kontrastmittelinjektionen und Biopsien, und die Entscheidung über therapeutische Maßnah-

167 Diese Einwilligung erfasst allerdings nicht die Weiterverwendung der Proben für Forschungszwecke.

168 Der Hessische Datenschutzbeauftragte (2004), 33. Tätigkeitsbericht 2004, Wiesbaden, S. 82.

169 Laufs (1993), Rdnr. 519. 
Teil F: Materialgewinnung, -lagerung und -nutzung

men gehören zu den nicht delegationsfähigen Leistungen. Generell delegationsfähige Leistungen sind demgegenüber:

- einfache Laborleistungen

- physikalisch-medizinische Leistungen

- Dauerkatheterwechsel

- einfache Verbände.

Nicht alle Leistungen sind generell delegationsfähig oder -unfähig. Einfache Injektionen, Infusionen und Blutentnahmen sind zwar dem Verantwortungsbereich des Arztes zuzurechnen, können aber im Einzelfall entsprechend qualifizierten nichtärztlichen Mitarbeitern übertragen werden. Entscheidend ist, dass ein persönliches Tätigwerden des Arztes nach Art und Schwere des Krankheitsfalles oder des Eingriffes nicht erforderlich ist.

Ein Arzt muss jede zu delegierende Tätigkeit im Einzelnen anordnen. Diese Anordnung sollte in hinreichend kritischen Fällen wie z.B. einer Blutentnahme möglichst schriftlich erfolgen. Vor Delegation und nach Anordnung der Leistung hat der Arzt sich selbst darüber zu vergewissern, dass der nichtärztliche Mitarbeiter in den entsprechenden Techniken besonders ausgebildet ist und diese auch beherrscht. Dies ist notwendig, weil die im Einzelfall delegationsfähigen Leistungen nicht zum üblichen Tätigkeitsbereich nichtärztlicher Mitarbeiter gehören. Dieser beschränkt sich im Allgemeinen auf die Grund- und Behandlungspflege sowie auf etwaige Vorbereitungshandlungen. Der Arzt sollte neben seinen Kontrollmaßnahmen im Behandlungsblatt auch dokumentieren, warum er von davon ausgeht, dass der nichtärztliche Mitarbeiter die erforderlichen Techniken beherrscht. Sofern etwaige Zertifikate oder schriftliche Nachweise („Spritzenschein“) vorhanden sind, ist dies ebenfalls zu vermerken.

\section{F.1.2. Probenverwahrung und -verwendung}

Die existierenden Vorgaben hinsichtlich der Verwahrdauer von Proben sind derzeit unklar oder uneinheitlich und sollten im Interesse der Spender aus der rechtlichen Grauzone geführt werden. Zwar gibt es z.B. in \I2 Abs. I HKG, der auf $\int \mathrm{I} 2 \mathrm{Abs} .4$ HDSG verweist, die Anforderung, den Patienten über die Verarbeitung seiner Daten und über seine Rechte zu informieren. Darunter wird auch die Dauer der Aufbewahrung fallen. In der Praxis wird dieser Anforderung aber nur wenig entsprochen. ${ }^{170}$

Der Hessische Datenschutzbeauftragte vertritt die Ansicht, dass für Behandlungszwecke gewonnene und aufbewahrte Proben nach \I2 Abs. 3 HKG und \33 Abs. I und 2 HDSG lediglich für konkrete Forschungsvorhaben verwendet werden dürfen, und eine Weitergabe der Proben für allgemeine Forschungszwecke, d.h. die Integration in BMB dadurch nicht legitimierbar wird. ${ }^{171}$ Hinsichtlich der Nutzung für allgemeine Forschungszwecke sollte daher eine klare datenschutz-

170 So werden etwa in den Aufnahmeverträgen der hessischen Kliniken bisher keine spezifischen Informationen über die Gewinnung, Aufbewahrung und Verwendung der Proben getroffen; ebenda, S. 83.

171 Ebenda, S. 85. 
rechtliche Grundlage geschaffen werden, die eingedenk der Stellungnahme des Nationalen Ethikrates zum Aufbau und Betrieb von Biobanken vom März $2004^{172}$, der Vorschläge der Datenschutzbeauftragten von 200I ${ }^{173}$ und des Anforderungskatalogs des Hamburgischen Datenschutzbeauftragten ${ }^{174}$ sowie des Hessischen Datenschutzbeauftragten ${ }^{175}$ vor allem folgende Angaben verbindlich vorschreiben sollte:

- die für die Proben und Daten dauerhaft verantwortliche Stelle,

- Dauer, Art und Weise der Speicherung und Aufbewahrung (pseudonymisiert oder anonymisiert),

- Umfang der gespeicherten Daten,

- Kreis der Personen, die von den personenbezogenen, pseudonymisierten oder anonymisierten Daten und Proben jeweils Kenntnis erhalten können,

- bei pseudonymer Speicherung und Aufbewahrung die möglichen Anlässe für eine Reidentifizierung der Spender,

- Hinweis auf die Freiwilligkeit der Einwilligung und darauf, dass dem Betroffenen durch die Ablehnung der Einwilligung keine Nachteile entstehen,

- Hinweis auf das Recht jedes Spenders, die Einwilligung für die Zukunft zu widerrufen und eine Herausgabe oder Vernichtung seiner Probe zu verlangen,

- Informationen zur eventuellen Unterrichtung des Spenders über Forschungsergebnisse. ${ }^{176}$

Dabei wird in der Literatur betont, dass sich die formularmäßige Einwilligungserklärung auf diese Informationen beziehen und getrennt von Proben und Daten aufbewahrt werden muss, und dass angemessene organisatorisch-technische Datensicherheitsmaßnahmen erforderlich sind, die Daten und Proben gegen unberechtigte Zugriffe Dritter schützen. ${ }^{177}$ Bei besonders sensitiven Proben und Daten wird sogar an das Hinzuziehen eines schweigeverpflichteten Datentreuhänders gedacht. ${ }^{178}$

\section{F.2. Probenlagerung und -transport}

Grundsätzlich bestehen keine besonderen, BMB-spezifischen Vorschriften für die Lagerung und den Transport von Biomaterial. Es müssen dabei die Eigenheiten des zu lagernden Materials und der aktuelle Stand von Wissenschaft und Technik beachtet werden, was bedeutet, dass je nach Art des Biomaterials geeig-

172 Nationaler Ethikrat (2004), www.ethikrat.org, Biobanken für die Forschung, S. 35.

173 Vgl. Der Hessische Datenschutzbeauftragte (2001), 30. Tätigkeitsbericht 2001, Wiesbaden, Ziffer 27.14.

174 www.hamburg.datenschutz.de.

175 Der Hessische Datenschutzbeauftragte (2004), 33. Tätigkeitsbericht, a.a.0., S. 87.

176 Der Hessische Datenschutzbeauftragte (2004), S. 87 f.

177 Es handelt sich bei diesen Forderungen um einen Katalog, der bisher noch nicht realisiert wurde.

178 Der Hessische Datenschutzbeauftragte (2004), S. 88. 
nete Lager- und Transportbehältnisse bereitzuhalten sind, die eine Zerstörung, Verschlechterung oder Veränderung des Materials verhindern. Hierzu sollte in Zusammenarbeit mit Experten ein entsprechender Leitfaden bzw. ein Pflichtenheft erarbeitet werden, in dem für jedes Biomaterial die Lagerungs- und Transportbesonderheiten aufzunehmen sind, d.h. Temperatur, Lagermedium (z.B. Parafin, Flüssigstickstoff), maximale Nutzungszeiten an der Umgebungsluft usw. ${ }^{179}$ Für infektiöses Material gelten die Regelungen des Infektionsschutzgesetzes bei Arbeiten und Verkehr mit Krankheitserregern. Datenschutzaspekte sind insoweit zu berücksichtigen, als Proben nicht zusammen mit identifizierenden Daten, regelmäßig sogar nur anonymisiert, verschickt werden dürfen. Selbstverständlich muss in jedem Fall das Arztgeheimnis gewahrt werden.

Die Einhaltung der oben genannten technischen Anforderungen ist insbesondere dann von Bedeutung, wenn die BMB Biomaterial gegen Entgelt an Dritte weitergibt. Ist das Biomaterial aufgrund von Versäumnissen der BMB bei der Lagerung oder beim Transport nicht verwendbar, so ist sie insoweit zur Gewährleistung und gegebenenfalls zum Schadenersatz verpflichtet. Wird die Mangelhaftigkeit des Biomaterials rechtzeitig entdeckt, werden sich die Ansprüche des Empfängers im Wesentlichen auf eine Nachlieferung einwandfreien Biomaterials beschränken, sofern dies möglich ist. Nicht abzuschätzen sind indes etwaige Schadenersatzansprüche des Empfängers, wenn sich erst nach Monaten herausstellt, dass zwischenzeitlich erzielte Forschungsergebnisse keine Aussagekraft haben, weil das gelieferte Biomaterial mangelhaft war. In diesem Fall könnte der Empfänger einen Anspruch auf Ersatz seiner nutzlosen Forschungsaufwendungen, sprich Personalkosten, Materialkosten etc. haben.

\section{F.3. Welchen Aufbewahrungspflichten unterliegen die Proben in einer BMB?}

Hinsichtlich der bestehenden Aufbewahrungspflichten von Biomaterialien ist grundsätzlich zwischen Behandlungsproben und Forschungsproben zu unterscheiden.

\section{F.3.1. Behandlungsproben: Beispiel Hessen}

Der Hessische Datenschutzbeauftragte hat in seinem letzten Jahresbericht ${ }^{180}$ gefordert, dass künftig in Dienstanweisungen intern klar festgelegt sein sollte, wie lange Proben in Arztpraxen oder Krankenhäusern für Behandlungszwecke aufbewahrt werden. Grundlage für diese Forderung war eine von ihm vorgenommenen Untersuchung, in der sich die Praxis der Aufbewahrung von Proben „weitgehend“, die Regelung der Aufbewahrungsfristen aber nur sehr aufwändig ermitteln ließ. Dabei waren die Dauer der Aufbewahrung und deren Gründe nicht

179 Zu den Einzelheiten der insoweit maßgeblichen Vorgaben wird auf die Ergebnisse zum Teilprojekt 4 des BMBGesamtprojekts verwiesen.

180 www.datenschutz.hessen.de/Tb33/K05P14. 
immer nachvollziehbar. Generell konnte er feststellen, dass offenbar keine konkreten einheitlichen Empfehlungen der medizinischen Fachgesellschaften existieren bzw. diese in der Praxis nicht immer befolgt werden. Er stellt ausdrücklich fest:

„Es ist in erster Linie eine Frage der fachlichen Bewertung, wie lange eine Aufbewahrung von Proben in den jeweiligen Klinikbereichen aus medizinischen Gründen erforderlich ist."

Er fordert dann,

„in diesem Bereich künftig Umfang und Zweck der Verwendung der Daten und Proben mit den Patientinnen und Patienten konkret schriftlich zu vereinbaren. Dies gilt gerade auch im Hinblick auf die derzeit in der Praxis zum Teil vorgesehene, zeitlich unbegrenzte Aufbewahrung von Proben und auch im Hinblick auf immer wieder vorkommende Anfragen von Angehörigen bezüglich einer erneuten Analyse der Proben für ihre Behandlungszwecke."

In Hessen dürfen Behandlungsproben grundsätzlich zwar nur für die konkrete Behandlung eines Patienten verwendet werden, unter bestimmten Voraussetzungen können sie aber auch ohne dessen Einwilligung für konkrete Forschungsvorhaben durch in- und externe Forscher genutzt werden ( $\iiint_{1}$ I Abs. 3 HKG, 33 HDSG). Für allgemeine Forschungszwecke fordert der Hessische Landesdatenschutzbeauftragte demgegenüber, die schriftliche Einwilligung des Patienten in die Verwendung seiner Probe einzuholen:

„Es liegt im Interesse der Patienten und Ärzte, aber auch und gerade der Forschung, dass die künftige Verwendung von Proben für allgemeine Forschungszwecke mittels Einwilligungserklärungen aufeine klare und verlässliche rechtliche Grundlage gestellt und die Verfahrensweise klar strukturiert wird."

Im Hinblick auf die Frage der Aufbewahrungspflicht wird verlangt, dass die Einwilligung Angaben zu Dauer, Art und Weise der Speicherung bzw. Aufbewahrung (pseudonymisiert oder anonymisiert) enthält. Auch hier wird selbstredend erwartet, dass die Proben vor unberechtigten Zugriffen Dritter sicher geschützt sind und von den Behandlungsdaten getrennt und sicher pseudonymisiert werden

\section{F.3.2. Forschungsproben}

Bei ausschließlichen Forschungsproben ist der Spender grundsätzlich über die Dauer der Nutzung seiner Probe zu informieren und seine Einwilligung in diesen Zeithorizont einzuholen. Ausdrückliche gesetzliche Regelungen, wie lange ausschließlich zu Forschungszwecken gewonnene Proben aufbewahrt werden müssen oder können, gibt es nicht. Einen Anhalt könnte allenfalls das BDSG liefern, das für sämtliche private und öffentliche Forschungsprojekte gilt. Unabhängig davon können sich entgegenstehende Aufbewahrungspflichten aus anderen Gesetzen (AMG, MPG, TFG etc.) ergeben. Es ist im Einzelnen zu prü- 
Teil F: Materialgewinnung, -lagerung und -nutzung

fen, ob diese Normen tatsächlich auch eine Aufbewahrungspflicht hinsichtlich (nicht anonymisierter) Proben begründen.

Nach $\iint 20$ II, 35 II BDSG sind Daten zu löschen, wenn ihre Speicherung unzulässig ist bzw. wurde oder ihre Kenntnis für die Daten verarbeitende Stelle zur Aufgabenerfüllung nicht mehr erforderlich ist. Proben sind zwar selbst keine personenbezogene Daten gemäß \3 I BDSG, sie können jedoch als Datenträger angesehen werden, aus denen genetische oder sonstige medizinische Erkenntnisse in Form personenbezogener Daten abgeleitet werden können. Aus der Forderung nach Löschung personenbezogener Daten gemäß $\mathbb{\int} 20$ II, 35 II BDSG ließe sich demnach auch eine Forderung nach Vernichtung der Probe ableiten, da diese den einzigen Weg zur expliziten Löschung der in dem „Datenträger Probe“ enthaltenen Informationen darstellt.

Unzulässig ist zudem eine Speicherung von Daten, wenn der Verarbeitungszweck erreicht wurde, d.h. bei Forschungsprojekten in der Regel bei deren Be-

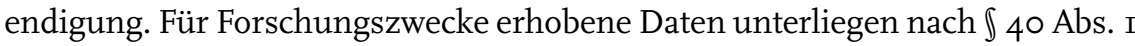
BDSG einer absoluten Zweckbindung. Sie dürfen daher für keine anderen als die fraglichen Forschungszwecke genutzt werden. Sofern die Einwilligungen des Spenders sich auf ein konkretes Forschungsprojekt beschränkt, können und müssen die Daten bzw. Datenträger (d.h. im vorliegenden Fall die Probe) vernichtet werden, wenn das Projekt abgeschlossen ist. Auch insofern können allerdings anderweitige Rechtsvorschriften (etwa aus den Krankenhausgesetzen der Länder) eine Legitimationsgrundlage für die Daten- und Probenweitergabe und -verwendung darstellen. Sofern eine zulässige Zweckänderung vorliegt, dürfen personenbezogene Daten und damit auch die Probe für den neuen Forschungszweck gespeichert werden. Allerdings bedarf es in diesem Fall entweder der Einwilligung des Spenders oder nach $\mathbb{\int}$ I4 Abs. 2 Nr. 9, 28 Abs. 6 Nr. 4 BDSG einer positiven Abwägung zugunsten der Interessen der Forschung gegenüber denen des Persönlichkeitsschutzes.

$\mathrm{Zu}$ beachten ist ferner, dass $₫ 40$ Abs. 2 BDSG die Anonymisierung, hilfsweise die Pseudonymisierung von Daten vorschreibt, sobald der Forschungszweck es gestattet. Dies bedeutet zunächst, dass die identifizierenden Merkmale (Name, Adresse, Geburtsdaten), die für die wissenschaftliche Auswertung nicht relevant sind, so früh wie möglich (also auch schon während des Forschungsprojektes) gelöscht oder zumindest verschleiert werden müssen. Dies gilt auch für Proben, die im Allgemeinen zunächst mit personenbezogenen Merkmalen verbunden sind. In der Regel sind diese personenbezogenen Daten getrennt von den Proben zu lagern. Soweit zum Teil die Auffassung vertreten wird, dass Körpermaterialien nie einer absoluten Anonymisierung zugeführt werden können, wird auf die Ausführungen in Abschnitt B.3.2.I.I. verwiesen. Sobald die Proben hinreichend anonymisiert im Sinne des $\ 3$ Abs. 6 BDSG sind (faktische Anonymisierung), können sie aus datenschutzrechtlicher Sicht unbefristet aufbewahrt werden. Aufgrund des biotechnologischen Fortschritts und der Weiterentwicklung der technischen Analysemöglichkeiten wird allerdings empfohlen, in regelmäßigen Zeitabständen die tatsächlichen Reidentifizierungsmöglichkeiten zu überprüfen. 
Liegt eine Einwilligung des Spenders in die Verwendung seiner Probe für allgemeine aber dennoch hinreichend präzise definierte Forschungsfragen vor, so kommt auch eine längere, eventuell sogar eine „ewige“ Aufbewahrung in Betracht. In diesen Fällen sind die Anforderungen an die Aufklärung und Informiertheit des Spenders und an die Bestimmtheit der Erklärung sehr hoch. Im Interesse des Persönlichkeitsschutzes bedarf es außerdem in solchen Fällen besonderer Sicherheitsmaßnahmen, zumal sich bei multifunktionalen Datenbeständen das Missbrauchsrisiko erhöht.

\section{F.4. Haftung bei Verstößen gegen Lagerungs-, Nutzungs- und Transportbestimmungen}

Grundsätzlich haftet der Betreiber einer BMB bei allen Verstößen, die im Rahmen ihres Geschäftsbetriebs vorkommen. Davon zu unterscheiden ist die Haftung der handelnden Organe, wenn diese einen Schaden verursacht haben und der Betreiber nachweisen kann, dass ihm kein Auswahl- und Organisationsverschulden (wie z.B. mangelnde Aufsicht) zur Last gelegt werden kann ( $\int 8$ 3 $\mathrm{I}$ BGB). Handelnde Organe können die Personen sein, deren sich der Betreiber zur Erfüllung seiner Aufgaben bedient. Bei der Probenentnahme sind dies in der Regel angestellte Ärzte, für andere Aufgaben sind es sonstige BMBMitarbeiter. Soweit ein Arzt involviert und das Arztrecht betroffen ist (z.B. Verletzung der ärztlichen Schweigepflicht, Behandlungsfehler etc.) haftet dieser für Verstöße gegen die ihm obliegenden Standespflichten neben dem Träger persönlich.

\section{F.5. Besteht ein gesetzliches Recht zur Nutzung von Biomaterialien?}

Ein gesetzliches Recht zur Nutzung der Biomaterialien hat nur der Eigentümer der jeweiligen Probe. Der Eigentümer der Probe ist in der Regel der Spender oder die BMB. Die BMB hat das Recht der Nutzung in dem Umfang, in dem es auf sie übertragen worden ist. Das Nutzungsrecht Dritter wird durch die Einwilligungserklärung definiert. Sofern die BMB berechtigt ist, die Materialien Dritten zur Verfügung zu stellen, haben diese im Verhältnis zur BMB ein vertragliches Nutzungsrecht, welches auch gegenüber dem Spender wirkt. Daneben ist noch ein gesetzliches Nutzungsrecht für staatliche Zwecke z.B. im Rahmen von staatsanwaltlichen Ermittlungsverfahren oder zur Abwehr einer schwerwiegenden Gefahr für die Bevölkerung möglich. Letzteres ist denkbar, wenn eine Probe z. B. für die Entwicklung eines Arzneimittels oder Impfstoffes benötigt wird, der zur Behandlung einer Epidemie dient.

Der Eigentümer der Biomaterialien hat grundsätzlich die Entscheidung über deren Verwendung. Ein vertragliches Nutzungsrecht kann jedoch auch Dritten gewährt werden, sofern damit nicht Rechte Dritter (Spender) oder gesetzliche 
Teil F: Materialgewinnung, -lagerung und -nutzung

Bestimmungen verletzt werden. Zu letzteren gehören bezüglich der aus den Materialien gewonnenen (bzw. der ihre Weitergabe begleitenden) Daten die Bestimmungen des BDSG. Die Nutzung der Materialien besteht in der Erhebung von Daten, deren Speicherung, Verarbeitung, Veränderung oder Verwendung für andere Zwecke.

\section{F.6. Durch wen können Nutzungsrechte entzogen bzw. übertragen werden?}

Die Nutzungsrechte an Biomaterialien können durch Vertrag grundsätzlich auf jede natürliche oder juristische Person übertragen werden. Unter Umständen kann ein Gläubiger mit einem entsprechenden Vollstreckungstitel gegen den Eigentümer in die Nutzungsrechte als geldwertes Vermögen die Zwangsvollstreckung betreiben. Durch die Zwangsvollstreckung würden die Nutzungsrechte dem Eigentümer entzogen werden. Sie werden damit nicht hinfällig, sondern gehen auf den Gläubiger bzw. Erwerber über. Zeitweise oder endgültig kann dem Eigentümer die Nutzung auch dann entzogen werden, wenn bei drohender oder bestehender Insolvenz ein Insolvenzverwalter die finanzielle Führung der BMB übernimmt. Er kann dann die Ausübung oder Verwertung der Nutzungsrechte nach seinen Vorstellungen und gegen den Willen des Eigentümers bestimmen. Die ordnungsgemäße Anwendung der Vorschriften des BDSG bleibt aber in allen Fällen zwingend.

Die Einwilligungserklärung ist immer durchschlagend, weil unabdingbar. Die Nutzungsrechte an einer Probe können durch den Spender bis hin zur Vernichtung entzogen werden ( $\iint 823$, I004 BGB). Voraussetzung hierfür ist, dass die konkrete Nutzungsart den Spender in seinem allgemeinen Persönlichkeitsrecht verletzt, also z.B. die konkrete Nutzungsart nicht von der Einwilligung des Spenders erfasst wird. Weitere Voraussetzung ist außerdem, dass die Körpermaterialien nicht anonymisiert sind. Im Falle der Anonymisierung der Probe ist das allgemeine Persönlichkeitsrecht des Spenders kaum noch betroffen. Selbst wenn man eine Verletzung des „biomateriellen Selbstbestimmungsrechts “ bejaht, wird eine Untersagungsverfügung faktisch nicht möglich sein, weil der Spender selbst schließlich nicht nachvollziehen kann, wer auf welche Art sein Körpermaterial nutzt. Er kann dann wegen der Verletzung seines Selbstbestimmungsrechts allenfalls einen Schadenersatzanspruch von der BMB verlangen.

Das Recht auf Nutzungsentzug durch den Spender schließt allerdings juristisch nicht aus, dass die BMB mit ihrem Probenbestand haftet. Wie unter E.3.2. bereits festgestellt, sind anonymisierte Proben nämlich grundsätzlich vollstreckungsfähig. Bei einer Nutzung durch Dritte kann die BMB in jedem Fall vertragliche Rechte, wie z.B. Rückrufrechte bei missbräuchlicher Nutzung einer Probe geltend machen. Entsprechende vertragliche Abmachungen sollten bei der Weitergabe getroffen werden, damit sich die BMB nicht Schadenersatzforderungen ausgesetzt sieht. 


\section{F.7. Zusammenfassung}

Bei der Lagerung und beim Transport von Proben sind Datenschutzaspekte insoweit zu berücksichtigen, als Proben nicht mit identifizierenden Daten zusammen verschickt werden dürfen (Erfordernis der Pseudonymisierung und Datentrennung). Die Proben sind aus Gründen des Datenschutzes getrennt von den personenbezogenen Daten aufzubewahren. Bei Verstößen gegen diese Bestimmungen haftet der Betreiber der BMB.

Die BMB hat das Recht der Nutzung in dem Umfang, in dem es auf sie übertragen worden ist. Etwaige Nutzungsrechte Dritter werden im Rahmen der Einwilligungserklärung definiert. Der Eigentümer hat grundsätzlich die Entscheidung über die Verwendung der Materialien. Dieses kann auf Dritte vertraglich übertragen werden (wobei datenschutzrechtliche Bestimmungen zu beachten sind). Außerdem können die Nutzungsrechte im Zuge einer Zwangsvollstreckung dem Eigentümer entzogen werden. Die Widerrufsrechte des Spenders bezüglich der Nutzung, die in der Einwilligungserklärung festgelegt wurden, werden von der Zwangsvollstreckung bei nicht anonymisierten Proben und Daten nicht berührt, da dann die Persönlichkeitsrechte unabdingbaren Vorrang vor Eigentums- und Nutzungsrechten genießen. 